type of experiment. We use a single rectangular plate with the proton beam running parallel to the long axis and a little above the plate. The beam, defined by stops, passes down the axis of a tube provided with a short interruption through which protons seattered by the gas in the camera can emerge to enter the plate.

A detailed account of the methods employed will shortly be submitted for publication in another place. It may be mentioned here that we find it a considerable advantage to use binocular microscopes and optical equipment of the highest quality to reduce the nervous strain on the observer to a minimum. Although the Ilford half-tone plate has proved very adequate for the work, it seems likely that it will be possible to develop special plates with superior characteristics for nuclear work.

\title{
THE VOCODER
}

\begin{abstract}
$\mathbf{A}^{\mathrm{T}}$ the World's Fairs in New York and San A Franciseo great interest was shown in the speech synthesizer shown in the Bell System exhibits. In the December number of the Bell Laboratories Record, H. Dudley, of their research department, describes this device, known as the 'vocoder'. The 'voder' is an offshoot of a more extensive system first demonstrated in its experimental stage several years ago. It first analysed spoken sounds and then used the information to control the synthesizing eircuit. As World's Fair displays were then under consideration it was seen that the synthesizer, manually controlled, could be made into a dramatic demonstration. Development was at first concentrated in this field, but when a successful Voder became assured, attention was shifted back to the broader and parent system and it was called the 'vocoder', since it operates on the principle of deriving voice codes to re-create the speech which it analyses. The analyser is at the left and the synthesizer at the right of the vocoder.
\end{abstract}

Electrical speech waves from a microphone are analysed for pitch by one channel and for spectrum by a group of channels. In the pitch analysis the fundamental frequency is called for simplicity the pitch. It is measured by a circuit containing a frequency discriminating network which obtains the frequency in a pure form, a frequency meter for counting, by more or less uniform pulses, the current reversals therein, and a filter for eliminating the actual speech frequencies but retaining a slowly changing current that is a direct measure of the pitch. The output current of the pitch channel is then a pitch-defining channel with its current approximately proportional to the pitch of the voiced sound and equal to zero for the unvoiced sounds.

There are ten spectrum-analysing channels, the first handling the frequency range 0-250 cycles and the other nine, the bands, 300 eycles wide, extending from 250 cycles to 2,950 cycles, a top frequency which is representative of commercial telephone eircuits. The operation of the analyser is illustrated by a group of oscillograms taken in analysing the sentence "She saw Mary". In the analyser the speech wave is the input and the eleven speechdefining signals are the output; in the synthesizer the eleven speech-defining signals are the imput and the speech wave the output. The relaxation oscillator is the source of the buzz, and the random noise circuit the source of the hiss. When a voiced sound is analysed a pitch current other than zero is received, with the result that the buzz is set for the current pitch by the "pitch control" on the relaxation oscillator; also, the relay marked "energy source switch" operates, switching from the hiss source to the buzz source. From the loud-speaker comes speech approximately the same in pitch and in spectrum as the original.

The possibilities of the Vocoder were recently demonstrated by the author before the Acoustical Society of America and before the New York Electrical Society. In these demonstrations comparison is first made between direct speech and the best recreation that the apparatus could make. Then by manipulation of dials and switches, speech is modified in various ways. Normal speech becomes a throaty whisper when the hiss is substituted for the buzz. Although the hiss is relatively faint, it is shown to be essential for discrimination between words like 'church' and 'shirts'.

Ordinarily the re-created pitch moves up and down with that of the original. If variation is prevented, the re-created speech is a monotone like a chant. When the relative variation is cut in half, the voice seems flat and dragging; when the swings are twice normal, the voice seems more brilliant; when four times normal it sounds febrile and unnatural. The controls can be reversed, so that high becomes low ; the tune of a song is then unrecognizable, and speech has some of the lilting characteristics of Scandinavian tongues. By appropriate setting of the basic pitch, the voice may be anything from a low bass to a high soprano. If the basic pitch is set to maintain a constant ratio of, say, 5 to 4 to the original, this is a 'major third' higher and harmonizes with the original. In two-part harmony, the demonstrator can then sing a duet with himself. Connecting a spare synthesizer for a 3 to 4 ratio he can sing one part in a trio, the others being taken by his electrical doubles. Finally, with the basic pitch control of the apparatus, he becomes a father reprimanding his daughter, then the girl herself, and then becomes the grandfather interceding for the child. Keeping careful time with the puffs of a locomotive, the demonstrator can make the locomotive puff intelligibly "We're--starting - slowly-faster, faster, faster" as the puffs come closer together. A particularly striking effect is that of singing with an organ to supply the tones. Although the words may be spoken, the demonstrator usually sings them to hold the rhythm. It makes no difference whether his voice is melodious or not; the tonal quality comes only from the musical source.

The engineering possibilities which may grow out of the application of the principles employed in this device are hard to predict at the present time. The speech-defining currents, however, do have features of simplicity and inaudibility which may open the way to new types of privacy systems or to a reduction in the range required for the transmission of intelligible telephonic speech. 